\title{
A REFINEMENT OF HÖLDER'S INTEGRAL INEQUALITY
}

\author{
ZHENG LIU
}

\begin{abstract}
The purpose of this note is to show that there is monotonic continuous function $p(t)$ such that

$$
\int_{a}^{b}\left(\prod_{i=1}^{n} f_{i}(x)\right) d x \leq p(t) \leq \prod_{i=1}^{n}\left(\int_{a}^{b} f_{i}^{r_{i}}(x) d x\right)^{\frac{1}{r_{i}}}
$$

where $f_{1}, f_{2}, \ldots, f_{n}$ are real positive continuous functions on $[a, b]$ and $r_{1}, r_{2}, \ldots, r_{n}$ are real positive numbers with $\sum_{i=1}^{n} \frac{1}{r_{i}}=1$.
\end{abstract}

It is well known that if $f_{1}, f_{2}, \ldots, f_{n}$ are real positive continuous functions on $[a, b]$ and $r_{1}, r_{2}, \ldots, r_{n}$ are real positive numbers with $\sum_{i=1}^{n} \frac{1}{r_{i}}=1$ then we have the Hölder's integral inequality

$$
\int_{a}^{b}\left(\prod_{i=1}^{n} f_{i}(x)\right) d x \leq \prod_{i=1}^{n}\left(\int_{a}^{b} f_{i}^{r_{i}}(x) d x\right)^{\frac{1}{r_{i}}}
$$

with equality holding if and only if $f_{1}^{r_{1}}, f_{2}^{r_{2}}, \ldots, f_{n}^{r_{n}}$ are effectively proportional.

Our aim is to give a continuous strictly increasing function $p(t)$ on $[0,1]$ such that

$$
\int_{a}^{b}\left(\prod_{i=1}^{n} f_{i}(x)\right) d x \leq p(t) \leq \prod_{i=1}^{n}\left(\int_{a}^{b} f_{i}^{r_{i}}(x) d x\right)^{\frac{1}{r_{i}}}
$$

for all $t \in[0,1]$.

Lemma.([1]) Let $f$ and $g$ be increasing functions on $[0,+\infty)$. Let $v$ and $h$ be nonnegative measurable functions, and let $a$ and $b, a<b$, be real numbers. Then

$$
\int_{a}^{b} f(v(t)) g(v(t)) h(t) d t \int_{a}^{b} h(t) d t \geq \int_{a}^{b} f(v(t)) h(t) d t \int_{a}^{b} g(v(t)) h(t) d t .
$$

Remark. By Theorem 10 of 2.5 in [2], we see that equality holds in (2) if and only if $v(t)$ is a constant.

Received July 1, 2002.

2000 Mathematics Subject Classification. 26D15.

Key words and phrases. Hölder's integral inequality, refinement, strictly increasing function. 
Theorem. Let $f_{1}, f_{2}, \ldots, f_{n}$ be real positive continuous functions on $[a, b]$. Let $r_{1}$, $r_{2}, \ldots, r_{n}$ be real positive numbers with $\sum_{i=1}^{n} \frac{1}{r_{i}}=1$.

Define a function $p$ by

$$
p(t)=\prod_{k=1}^{n}\left\{\int_{a}^{b}\left[\prod_{i=1}^{n} f_{i}(x)\right]^{1-t}\left[f_{k}^{r_{k}}(x)\right]^{t} d x\right\}^{\frac{1}{r_{k}}}, \quad t \in(-\infty,+\infty) .
$$

Then $p^{\prime}(t) \geq 0$ for $t>0, p^{\prime}(t) \leq 0$ for $t<0$, and $p^{\prime}(t)=0$ if and only if $t=0$ or $f_{1}^{r_{1}}$, $f_{2}^{r_{2}}, \ldots, f_{n}^{r_{n}}$ are effectively proportional.

Proof. It is easy to find that for each $x \in[a, b]$ we have

$$
\sum_{k=1}^{n} \frac{1}{r_{k}} \log \frac{f_{k}^{r_{k}}(x)}{\prod_{i=1}^{n} f_{i}(x)}=\sum_{k=1}^{n} \frac{1}{r_{k}}\left[r_{k} \log f_{k}(x)-\sum_{i=1}^{n} \log f_{i}(x)\right]=0
$$

and hence

$$
\sum_{k=1}^{n} \frac{1}{r_{k}} \int_{a}^{b}\left(\prod_{i=1}^{n} f_{i}(x)\right) \log \frac{f_{k}^{r_{k}}(x)}{\prod_{i=1}^{n} f_{i}(x)} d x=0
$$

Let $g(x)=\prod_{i=1}^{n} f_{i}(x), h_{k}(x)=\frac{f_{k}^{r_{k}}(x)}{g(x)}, k=1,2, \ldots, n$.

Then $g(x)$ and $h_{1}(x), h_{2}(x), \ldots, h_{n}(x)$ are all real positive continuous functions on $[a, b]$ and we can write $(3)$ as

$$
p(t)=\prod_{k=1}^{n}\left[\int_{a}^{b} g(x) h_{k}^{t}(x) d x\right]^{\frac{1}{r_{k}}} .
$$

Let $P(t)=\log p(t)$. Observe that (4) can write as

$$
\sum_{k=1}^{n} \frac{1}{r_{k}} \int_{a}^{b} g(x) \log h_{k}(x) d x=0
$$

We obtain

$$
\begin{aligned}
P^{\prime}(t) & =\frac{p^{\prime}(t)}{p(t)}=\sum_{k=1}^{n} \frac{1}{r_{k}} \frac{\int_{a}^{b} g(x) h_{k}^{t}(x) \log h_{k}(x) d x}{\int_{a}^{b} g(x) h_{k}^{t}(x) d x} \\
& =\sum_{k=1}^{n} \frac{1}{r_{k}} \frac{\int_{a}^{b} g(x) h_{k}^{t}(x) \log h_{k}(x) d x}{\int_{a}^{b} g(x) h_{k}^{t}(x) d x}-\sum_{k=1}^{n} \frac{1}{r_{k}} \frac{\int_{a}^{b} g(x) \log h_{k}(x) d x}{\int_{a}^{b} g(x) d x} \\
& =\sum_{k=1}^{n} \frac{1}{r_{k}} \frac{\int_{a}^{b} g(x) h_{k}^{t}(x) \log h_{k}(x) d x \int_{a}^{b} g(x) d x-\int_{a}^{b} g(x) h_{k}^{t}(x) d x \int_{a}^{b} g(x) \log h_{k}(x) d x}{\int_{a}^{b} g(x) d x \int_{a}^{b} g(x) h_{k}^{t}(x) d x}
\end{aligned}
$$

Consequently, the results follow from the lemma and its remark. 
Corollary. Let $f_{1}, f_{2}, \ldots, f_{n}$ be real positive continuous functions on $[a, b]$. Let $r_{1}$, $r_{2}, \ldots, r_{n}$ be real positive numbers with $\sum_{i=1}^{n} \frac{1}{r_{i}}=1$. Then the function $p$ defined by (3) has the following properties:

(i) $p(t)$ is continuous strictly increasing for $t \geq 0$ and continuous strictly decreasing for $t<0$ or a constant.

(ii) $p(t)$ is a constant if and only if $f_{1}^{r_{1}}, f_{2}^{r_{2}}, \ldots, f_{n}^{r_{n}}$ are effectively proportional.

(iii) $p(0)=\int_{a}^{b}\left(\prod_{i=1}^{n} f_{i}(x)\right) d x$ and $p(1)=\prod_{i=1}^{n}\left(\int_{a}^{b} f_{i}^{r_{i}}(x) d x\right)^{\frac{1}{r_{i}}}$.

(iv) The inequalities (1) are valid for all $t \in[0,1]$ with equalities holding if and only if $f_{1}^{r_{1}}, f_{2}^{r_{2}}, \ldots, f_{n}^{r_{n}}$ are effectively proportional.

\section{References}

[1] D. S. Mitrinovic, J. E. Pecărić and A. M. Fink, Classical and New Inequalities in Analysis, Kluwer Academic Publishers, Dordrecht-Boston-London, 1993.

[2] D. S. Mitrinovic, Analytic Inequalities, Springer Verlag, Berlin-Heidelberg-New York, 1970.

Institute of Applied Mathematics, Faculty of Science, Anshan University of Science and Technology, Anshan 114044, Liaoning, P. R. China. 